\title{
L'État ottoman et la guerre de Trente Ans, les dommages à long terme de la neutralité
}

The Ottoman State and the Thirty Years War, the long-term damage of neutrality

Jean-Louis Bacqué-Grammont

\section{OpenEdition}

\section{Journals}

Édition électronique

URL : https://journals.openedition.org/ceb/3982

DOI : $10.4000 /$ ceb.3982

ISSN : 2261-4184

\section{Éditeur}

INALCO

Édition imprimée

Pagination : 267-270

ISBN : 978-2-85831-205-4

ISSN : 0290-7402

\section{Référence électronique}

Jean-Louis Bacqué-Grammont, "L'État ottoman et la guerre de Trente Ans, les dommages à long terme de la neutralité », Cahiers balkaniques [En ligne], 41 | 2013, mis en ligne le 19 mai 2013, consulté le 06 juillet 2021. URL : http://journals.openedition.org/ceb/3982 ; DOI : https://doi.org/10.4000/ceb. 3982

Ce document a été généré automatiquement le 6 juillet 2021.

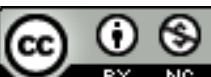

Cahiers balkaniques est mis à disposition selon les termes de la Licence Creative Commons Attribution - Pas d'Utilisation Commerciale 4.0 International. 


\section{L'État ottoman et la guerre de Trente Ans, les dommages à long terme de la neutralité}

The Ottoman State and the Thirty Years War, the long-term damage of neutrality

Jean-Louis Bacqué-Grammont

\section{NOTE DE L'AUTEUR}

La présente étude s'inscrit dans le cadre du programme de recherche sur « Histoire et sciences auxiliaires de l'histoire ottomane ", commun à l'Unité mixte de Recherche 7192 du Centre National de la Recherche scientifique (Paris) et à l'Institut français d'Études Anatoliennes d'Istanbul, et dirigé par l'auteur de ces lignes. Nous reprenons ici, en les développant, certains points abordés dans notre communication « Kâtip Çelebi, Evliya Çelebi : Ortak Bir Kaygının Kavramlaşması » [Kâtip Çelebî, Evliyâ Çelebî et la conceptualisation d'une commune inquiétude], présentée au Uluslararası Kâtip Çelebi Sempozyumu ve Piri Reis'ten Kâtip Çelebi'ye Osmanlı Dünya'ya Bakışı [Symposium international sur Kâtib Çelebî. Le regard des Ottomans sur le monde de Pîrî Re'îs à Kâtib Çelebî], ministère de la Culture et du Tourisme de la République de Turquie et Centre de recherche sur les civilisations de l'Université de Bahçeşehir, Ankara, 19-20 avril 2009, sous presse dans les Actes. Une version turque abrégée de ce texte a été publiée sous le titre "Osmanlı Devleti ve Otuz Yıl Savaşları. Tarafsızlığın Uzun Vadede Zararları", I. Uluslararası LAÜ Tarih Kongresi. Osmanlı ve Cumhuriyet Dönemi Türk Tarihi. 1rst International EUL History Congress. Turkish History of Ottoman and Republic Period. 6-9 Nisan 2011. Bildiri Kitab1, Lefke, Université Européenne, pp. 119-123.

1 Les troupes turques sont pleines d'ardeur et d'intrépidité, le feu de l'infanterie ne les arrête pas ; la baïonnette seule réprime leur fougue. (...) Ce sont des troupes légères de 
la meilleure espèce. Elles attaquent vivement, sans ordre, sans harmonie, sans plan combiné relativement au terrain ou à la disposition de l'ennemi. Elles l'environnent et fondent sur lui de toutes parts. (...) Un corps est repoussé, un autre le relève et n'a pas plus de succès. Ils entraînent en fuyant ceux qui accourent après eux. La cavalerie et l'infanterie se nuisent et se confondent; les attaques sont plus faibles, la confusion devient générale et conduit à une retraite presque aussi vive que le premier choc $^{1}$.

Dans un article désormais classique, Halil İnalcık a souligné la difficulté avec laquelle, au cours de la «longue guerre » contre les Impériaux (1593-1606), l'armée ottomane intégra dans ses rangs des unités de mousquetaires, récemment apparues, et peina à les employer de manière aussi efficace que ses adversaires chrétiens ${ }^{2}$. Dans le même ouvrage, V. J. Parry a montré à quel point ces derniers avaient amélioré leur tactique, jusqu'à en élaborer certaines, spécifiques, pour affronter des Ottomans qui, eux, n'avaient guère perfectionné la leur depuis le temps de Soliman le Magnifique. À ces considérations, il convient d'en ajouter une, non moins importante. Il nous semble qu'on n'a pas accordé l'attention nécessaire au fait que non seulement les Ottomans demeurèrent complètement étrangers à la guerre de Trente Ans, mais qu'ils n'en tirèrent aucune des leçons militaires qui s'imposaient, au contraire de la plupart des armées d'Europe. Armement, tactique, tout changea alors. À la bataille de Breitenfeld, en 1631, la cadence de tir deux fois plus rapide et les «canons de cuir bouilli » de Gustave-Adolphe de Suède, utilisables par des cavaliers, firent une hécatombe des troupes du comte de Tilly : 13000 tués contre 3000 ). On allait bientôt voir les fameux tercios espagnols, réputés jusqu'alors invincibles, mis à mal par les hakkapélites, cavaliers finlandais faisant un usage méthodique de leurs armes à feu, puis, finalement, par l'habileté manœuvrière du prince de Condé à la bataille de Rocroi, le 19 mai 1643. Mais les troupes et les arsenaux de l'Empereur Ferdinand II ne tardèrent pas à relever les défis techniques et tactiques des Suédois. En définitive, si les Habsbourg d'Autriche et d'Espagne ne gagnèrent pas la guerre de Trente Ans, du moins l'expérience qu'ils y avaient acquise leur conféra à partir de la bataille de Saint-Gothard une supériorité évidente et durable sur les Ottomans qui, de leur côté, combattaient toujours comme à la veille de la paix de Zsitvatorok, près de soixante années plus tôt.

3 La « longue guerre » entre les Ottomans et les Impériaux marque, en effet, une étape importante dans les affrontements entre ces deux puissances. Jusqu'alors, la suprématie sur les champs de bataille revenait sans aucun doute aux Ottomans, supérieurs de beaucoup en nombre d'hommes, et dont la cavalerie des sipahi aussi bien que l'infanterie des janissaires constituaient des unités d'une valeur militaire difficile à égaler. Discipline, mobilité et endurance faisaient en outre leur force et devaient la faire longtemps encore. Mais les choses changèrent dès le début de cette "longue guerre » qui survint après plusieurs décennies d'une paix relative. Les treize années de ce conflit indécis, épuisant et ruineux pour chacune des parties, laissèrent apparaître pour la première fois un équilibre des forces en présence. Celles-ci finirent par conclure, en 1606, une paix consacrant une telle situation: pour la première fois, l'Empereur n'était plus le tributaire du Padişah, mais son égal. Soixante années de paix devaient suivre.

Que s'était-il passé ? Sans être un expert de l'histoire de l'armement, on peut discerner que le dernier quart du $\mathrm{XvI}^{\mathrm{e}}$ siècle est marqué en Europe par une évolution rapide des armes à feu de tous calibres. Les nouvelles couleuvrines du maître Gregor Löffler d'Innsbruck introduites en Angleterre par son neveu Adam Dreyling, jouèrent un rôle 
notable dans l'échec de l'Invincible Armada en 1588: elles pouvaient tirer un coup toutes les deux minutes - contre dix minutes pour les canons espagnols -, elles avaient une plus longue portée et une force de pénétration supérieure, comme de récentes expériences ont permis de le vérifier. C'est ainsi que quelques corsaires anglais purent harceler les 130 galions du roi Philippe II sans crainte pour eux-mêmes. C'est à la même époque que l'arquebuse laissa la place au mousquet, plus léger et tirant des balles de plus fort calibre à plus longue portée.

Dès le début de la « longue guerre », les Ottomans apprirent à leurs dépens les effets de ces nouvelles armes et cherchèrent naturellement à s'en doter à leur tour. C'est sans doute dans ce contexte que se trouve l'explication de leur attitude étonnamment bienveillante et patiente à l'égard du fameux « régiment français» au tournant du $\mathrm{XVII}^{\mathrm{e}}$ siècle. Ces mercenaires lorrains, wallons et autres qui n'avaient de français que leur langue commune, avaient été recrutés pour les besoins de la guerre en Hongrie par l'Empereur Rodolphe II. Toutefois, ne recevant pas leur solde, ils étaient passés au service du Padişah avec des conditions extrêmement favorables. Pour autant qu'on puisse en juger, ils se comportèrent de manière fort efficace partout où ils furent engagés. Mais les Ottomans auraient-ils supporté longtemps cette troupe de soudards d'une effroyable cruauté si elle n'avait été équipée des nouvelles armes? Or, on peut suivre leur trace pendant plus d'un quart de siècle, au moins jusqu'à la campagne de Podolie d'Osman II.

6 Cette fois-là, les Ottomans avaient réagi avec rapidité devant des développements technologiques imprévus. Mais la soixantaine d'années de non-belligérance avec l'Empire qui suivit la paix de Zsitvatorok devait changer complètement le rapport des forces. Certes, le Padişah n'avait aucune raison de s'engager dans la guerre de Trente Ans (1618-1648) qui ne concernait que des catholiques et des protestants. Mais n'y pas participer était une chose, n'en vouloir rien savoir, une autre. Si les Ottomans, longtemps à la pointe des progrès de l'armement et qui disposaient d'informateurs nombreux en Europe chrétienne cent ans plus tôt, avaient suivi avec la même attention que naguère les événements d'Allemagne dans la première moitié du XVII siècle, ils auraient constaté que tout changeait là-bas très rapidement, aussi bien les armes que la manière de s'en servir : artillerie de campagne, piques, baïonnettes, chevaux de frise, etc. Ignorants de la nouvelle manière de combattre, ils se trouvèrent soudain en 1664, à Saint-Gothard, face à des adversaires qui, eux, avaient savamment étudié la leur et savaient comment l'affronter. Pour résumer, jusqu'à la fin de l'époque médiévale, la pensée tactique des Européens, obnubilée par les récits chevaleresques, n'avait voulu connaître de vertu qu'à la charge de cavalerie. Après Damiette, Crécy, Poitiers et Azincourt cela avait naturellement mené à de nouveaux désastres face aux Ottomans : Nicopolis, Varna, Kosovo, Mohaç. Désormais, l'enveloppement par les ailes et la fuite simulée étaient des tactiques ottomanes bien connues de l'adversaire chrétien, mais aussi périmées que les charges de cavalerie de celui-ci. Or, refusant de voir que leur nombre ne faisait plus rien en face des nouvelles armes et des mouvements inattendus de l'adversaire, les Ottomans s'obstinèrent à ne rien changer dans leur manière de combattre ${ }^{3}$. Quand ils le firent, il était trop tard depuis longtemps. Un proverbe dit que la curiosité est un vilain défaut. On devrait ajouter que l'absence de curiosité est bien pire. C'est ce que nous avons tenté d'illustrer brièvement ici. 


\section{NOTES}

1. Louis-Félix Guynement de Kéralio (1731-1793), Histoire de la guerre entre la Russie et la Turquie et particulièrement de la campagne de MDCCLXIX, Saint-Pétersbourg, 1773, p. 100, cité par V.J. Parry, «La Manière de combattre », dans War, Technology and Society in the Middle East, éd. V. J. Parry et M. E. Yapp, Londres - New York - Toronto, Oxford University Press, 1975, p. 243.

2. "The Socio-Political Effects of the Diffusion of Fire-arms in the Middle East", op. cit, pp. 199-200.

3. On en trouvera une illustration particulièrement éloquente émanant d'un témoin visuel dans l'ouvrage de Faruk Bilici, Evliya Tchélébi. La guerre des Turcs. Récits de bataille extraits du Livre de Voyages, collection Bibliothèque turque, Arles, Actes-Sud - Sindbad, 2000.

\section{RÉSUMÉS}

La guerre qui opposa Ottomans et Impériaux de 1593 à 1606 apparaît remarquable non seulement par sa longue durée, mais aussi par l'égalité des forces en présence : chacun des adversaires obtient tour à tour un avantage, mais aucun de ceux-ci n'est décisif. La paix sera donc recherchée avec un égal empressement par les deux parties. Lorsqu'elle prendra fin après plus d'un demisiècle, on verra les Ottomans perdre pied dès les premières batailles et, malgré leurs puissants moyens, ne plus parvenir à renouer avec leurs succès d'autrefois. Que s'était-il passé entre temps? Nous tenterons de souligner ce que leur coûta leur manque d'intérêt à l'égard de la guerre de Trente Ans (1618-1648) : après celle-ci, le nouvel armement, son emploi et l'art de la guerre, tout avait changé en Europe occidentale et il semble qu'on n'en savait rien sur les rives du Bosphore.

The war between Ottomans and Habsburgs from 1593 to 1606 looks noteworthy not only because it had been going on for many years, but also because they were equally matched forces: each side alternatively took the advantage over the other, but none of them was conclusive. In consequence, peace was searched with eagerness by both sides. When it finally ended more than half a century later, the Ottomans were in difficulty right from the first battles and, in spite of their great power, they could not find back their preceding successes. What had happened in the meantime? We shall attend here to underline the cost of their lack of interest towards the Thirty Years War (1618-1648): after that, new weapons and the ways of using them, everything in Western Europe had changed concerning the art of war and it seems that nothing of these new conditions was known on the shore of the Bosphorus. 
INDEX

Thèmes : Histoire

Keywords : weapons, modernization of military equipment, Ottoman/Habsburg War, History, seventeenth century

Index géographique : Empire ottoman

motsclesmk ОРУЖЈЕ, ОПРЕМА, ВОЈНА ОТОМАНСКАТА / ХАБСБУРГ, ОТОМАНСКАТА ИМПЕРИЈА, ИСТОРИЈА, СЕДУМНАЕСЕТИОТ ВЕК

motsclestr Silah, Silah modernizasyonu, Savaş Osmanlı / Habsburg, Osmanlı İmparatorluğu, Tarih

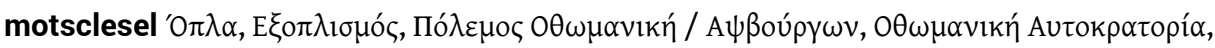

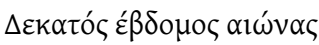

Mots-clés : Hakkapélites, armement, Longue guerre, modernisation de l'armement, Tercio, Guerre Ottomans/Habsburgs

Index chronologique : Empire ottoman 